\title{
Autonomic Disorders in Multiple Sclerosis
}

\author{
E. Lensch and W. H. Jost \\ Department of Neurology, Deutsche Klinik für Diagnostik, Aukammallee 33, 65191 Wiesbaden, Germany
}

Correspondence should be addressed to W. H. Jost, jost.neuro@dkd-wiesbaden.de

Received 31 October 2010; Accepted 24 February 2011

Academic Editor: Ronald Tuma

Copyright () 2011 E. Lensch and W. H. Jost. This is an open access article distributed under the Creative Commons Attribution License, which permits unrestricted use, distribution, and reproduction in any medium, provided the original work is properly cited.

\begin{abstract}
Multiple sclerosis is an inflammatory disease leading to disseminated lesions of the central nervous system resulting in both somatomotor and autonomic disturbances. These involve the central centers of the autonomic nervous system, as well as the automatic control and pathway systems. All autonomic functions may be disordered individually or in combined form. There is no other disease with a clinical picture so multifaceted. Besides cardiovascular dysfunctions disorders of bladder and rectum have become apparent. Somatomotor and autonomic disturbances occur with similar frequency; however the focused exam often heavily favors somatomotor symptoms. Autonomic disturbances should primarily be taken into account on history taking and clinical examination. Individual diagnosis and treatment is a secondary feature. Impairments of the autonomic nervous systems in multiple sclerosis are frequently overlooked.
\end{abstract}

\section{Introduction}

Although there are obvious disturbances of the somatic and autonomic nervous system in disseminated CNS-disease, in the diagnosis and therapy of multiple sclerosis there is less focus on the autonomic nervous system than on the somatic nervous system. Vegetative symptoms are mostly recorded without classifying them to a morphologic lesion as is being done with motor and sensory deficits. By comparison with somatic symptoms, autonomic disturbances are widely disregarded in the literature as well as in scientific research. A great number of autonomic dysfunctions are not being attended to by neurologists but by other medical specialists. This leads to deficits in the diagnostic workup and treatment of the illness, as patient management is neither centralized nor coordinated. It should principally be the neurologist's responsibility to guide diagnostics and to include his reflections and observations in the treatment that has been recommended and carried out.

Any vegetative function can be impaired due to the dissemination of the lesions. Reference in this respect is, of course, made to the autonomic nervous system (Figure 1). Since the lesion is rarely affecting one area alone, we frequently see a complex constellation of symptoms that can be both, progressing as well as regressing. This holds particularly true for myelin, the pathways of which may be afflicted, and on the other side for the sympathetic and parasympathetic systems that may be disrupted in varying degrees.

Symptoms involve the entire autonomic nervous system, especially the urogenital tract, gastrointestinal tract, and the cardiovascular system.

\section{Dysfunctions of the Bladder}

Regulation of urinary storage and urinary evacuation is highly complex involving many structures of the central nervous system. This is why urologic symptoms are frequent and typical sequelae of inflammation-induced lesions of the central nervous system. In 5-9\% of patients, urologic symptoms represent the first manifestation of multiple sclerosis $[1,2]$. In later stages of this illness, approximately $80-90^{+} \%$ of all patients are dealing with bladder dysfunction [3-6]. In theory, bladder dysfunction may be episodic with intermittent acute symptoms, which may complicate the diagnostic process.

The topographically disseminated pathologic activity of multiple sclerosis (MS) does damage to various centers (predominantly the frontal and pontine micturition center and 


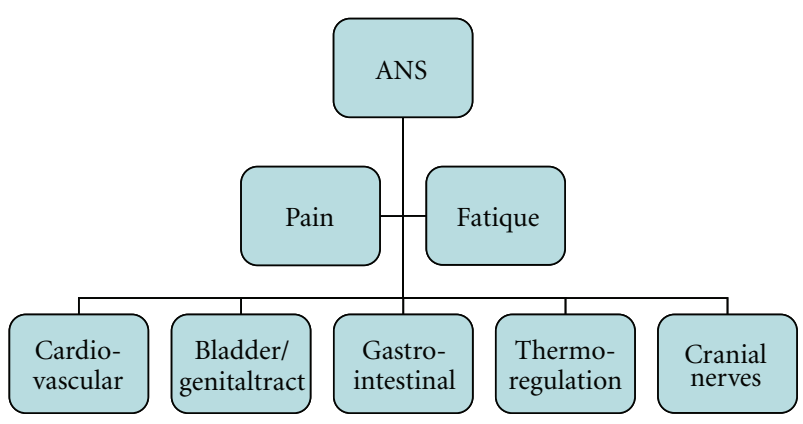

FIgURE 1: Focal points of the autonomic nervous system.

the spinal cord) aside from other structures of the complex control loop of neurogenic bladder regulation. Lesions above the pontine micturition center will lead to reduced inhibition and, consequently, to hyperactivity of the detrusor muscle; meaning the urge to urinate in the absence of adequate bladder filling. Spinal lesions in the cervical and thoracic marrow will also result in diminished central inhibition via damage to the sensory afferences and to the pyramidal tract. Lesions in this area result not only in hyperactivity of the detrusor but also in disrupted coordination between detrusor contractions and sphincter relaxation (detrusor sphincter dyssynergy). In addition, damage to the pyramidal tracts will generate spasticity of the striated sphincters. Detrusor hypoactivity is considerably rarer and frequently secondary in nature [7]. Overall, the correlation between the character of bladder dysfunction and the pattern of lesions seen on magnetic resonance imaging (MRI) appears to be rather poor; on the other hand the correlation between the degree of bladder dysfunction and the degree of disability and pyramidal malfunction was corroborated by quite a few authors [7-9].

\section{Neurologic Diagnosis}

The diagnosis of bladder dysfunctions in patients with multiple sclerosis is frequently drawn from accessory remarks (ancillary information) on history taking. The examiner should always and explicitly inquire about difficulties associated with urination. Polyuria is often the first symptom patients mention. In addition, they complain of newonset nocturia. Increased nighttime micturitions are often dismissed. Contrarily urge symptoms, which are experienced in the further course of disease, are usually reported spontaneously against the background of imminent or rare urinary incontinence. Urinary tract infections do not play a major symptom-defining role at this stage of bladder dysfunction.

Difficulty voiding is a rare urologic first symptom of multiple sclerosis. It is generally reported at once and should be diagnosed and treated without delay to prevent acute complications as well as detrusor and kidney alterations before their manifestation as secondary long-term sequelae.

In later stages of the disease, there is clinical evidence of both difficulty voiding as well as insufficient storage. While increases in urinary frequency and incontinence primarily lead to social restrictions, increased residual urine causes concomitant urinary tract infections. Contrary to traumatic paraplegia, lesions of the upper urinary tract are significantly less common in female patients with MS [10], but they never cease to be a possible complication in the disease progression [11]. Aggravated bladder dysfunction is mostly associated with gradually progressing dysfunction of sensory and particularly motor pathways to the lower limbs.

Studying bladder filling and evacuation by video urodynamics permits the clearest analysis of impaired bladder function. The parameters monitored include internal vesicular pressure, intrarectal pressure, voided volume, urinary flow and muscular activity of the pelvic floor. At the onset of voiding (i.e., detrusor contraction) MS patients will typically present with a higher muscle tone in the pelvic floor, which is passed on to the vesical sphincter muscle, thereby impeding the process of urination. This finding has been termed detrusor sphincter dyssynergy (DSD). Sonographic determination of residual urine is insufficient to conclusively determine the exact nature of the dysreflexia; however residual volume is the most relevant clinical factor in this classification. It is therefore appropriate for an overall check on the effect and on the necessity of treatment.

The validity of neurophysiologic tests is limited. Sensory evoked potentials (SEPs) of the pudendal nerve are helpful in objectifying the extent of afferent damage. Electromyography (EMG) of the striated sphincters may provide clues to differential diagnosis.

There may be additional bladder dysfunctions apart from MS typical DSD, and these have to be excluded in terms of differential diagnosis. This applies to prostatic hyperplasia in older male subjects or symptoms of stress incontinence present in older women.

\section{Treatment of Bladder Dysfunctions}

Drug therapy of DSD is aimed at two aspects: suppression of the urge to pass water or the overly frequent reflexive voiding of the bladder, and secondarily the relaxation of the sphincter tonus to improve urinary output.

The inhibition of detrusor hyperactivity is accomplished by drug-induced suppression of the parasympathetic innervation - primarily by the application of anticholinergic agents. In case of unsatisfactory effect, local injection of botulinum toxin might be appropriate for some patients $[12,13]$. Patients with mild urge incontinence can benefit from the anticholinergic action of tricyclic antidepressants. Initiation of treatment as described bears the risk of accumulating residual urine; therefore, it is necessary to monitor residual volume during the initial phases of treatment. This also applies to other parasympatholytic or sympathomimetic medication. Concomitant treatment of the drainage disorder is required at the latest when residual urine has come up to more than $100 \mathrm{~mL}$. Antispasmodics, used previously in the course of treatment for symptoms not relating to problems with the bladder become the agent of choice. Bladder trouble, in some cases, may be the only indication for their long-term application. Despite differentiated medication, we cannot 
always prevent stagnant urine from worsening or preserve the ability to retain urine. These two problems can usually be resolved by intermittent self-catheterization. There are physical prerequisites to that, namely, adequate manual skills and a fairly low adductor tonus. An indwelling catheter is the provisionary solution for patients who do not meet these requirements or who are likely to fail in self-catheterization for whatever reason.

Sacral neuromodulation [14] has been successful in only a few MS patients and is therefore not recommended.

Physiotherapeutic approaches and rehabilitation programs [15] are suitable to enhance pharmacotherapy. Training of the muscular system of the pelvic floor and biofeedback methods can be contributory to maintaining continence and in supporting relaxation of the external sphincter muscle (to avoid triggers for instance).

4.1. Sexual Dysfunctions. The most thoroughly researched solitary sexual problem is erectile dysfunction in males suffering from MS. In neurological literature, including older publications, the incidence is rated approximately: 60\% [1619]. A sexual science study on MS patients in Northern Germany revealed poor erections in $42 \%$ of these men [20]. To evaluate these findings, data should be incorporated with regard to similar complaints prior to the onset of disease or those of a control group.

The finding of reduced sexual arousal corresponding with DSM-IV in women suffering from MS has not been systematically covered in older literature. Beier's aforementioned retrospective inquiry identified a prevalence of $18 \%$. Dyspareunia was specified in this study for $51 \%$ of male and $22 \%$ of female patients. Neurologic surveys with considerably smaller groups reported anorgasmy or reduced ability to experience an orgasm in $64 \%$ of men and $58 \%$ or $12 \%$ of women $[4,21]$. Libido disorders or decreased sexual appetence (Diagnostic and Statistical Manual of Mental Disorders Nomenclature) seem to be more common in affected men and is reported by approximately one-third of this population.

The correlation between disturbances of pathways, the overall degree of impairment and bladder dysfunctions with sexual dysfunction was investigated by several researchers. Sexual dysfunctions are exacerbated in the course of the disease thus exhibiting the typical picture of other functional impairments in MS patients. Moreover, in men it is common to see impotency is largely associated with bladder dysfunction and lesions of the pyramidal tract [22] Women apparently do not present with sexual dysfunction linked with bladder dysfunction to the same extent [23]. Diminished lubrication and disturbed sensation in the genital region on the other hand, is frequently associated with sexual dysfunctions in female MS patients $[19,24]$. A more recent study on women suffering from MS disclosed that there is no correlation between sexual dysfunction and a pathologic sympathetic skin response on sensory testing in the genital region [25].

The communication of the diagnosis of MS has a farreaching effect. This is corroborated by the fact that sexual contentment, of both patients and their partners, ebbed substantially after the diagnosis had been conveyed to them. This does not appear to be related to the true degree of organically explainable dysfunctions [20].

Sexual dysfunctions are considerably more frequently multifactorial in nature than bladder dysfunctions, and may be substantially determined by nonorganic causes. Historytaking is encumbered by the great variety of symptoms, aside from physicians' and patients' insecurity or reluctance to deal with the issue. Comparable data are thus hardly available.

The diagnostic procedure for sexual dysfunctions in MS patients varies from the comparable situation of previously healthy subjects inasmuch that extended neurologic diagnosis by exclusion can usually be neglected. Additional urologic and gynecologic evaluation, is called for to rule out nonneurological causes. Disturbed sensibility can to some degree be objectively recorded by pudendal SSEP, and sympathetic involvement by penile sympathetic skin response (SSR).

Erectile dysfunction can most easily be subjected to treatment. Earlier trials with pharmaceutical agents have faded into the background after the introduction of phosphodiesterase inhibitors. Up to $90 \%$ of the patients related a positive effect [26].

Intracavernous prostaglandin injections and vacuum pumps are reserved to exceptional cases. There is no ascertained drug treatment of sexual dysfunctions in female MS patients. The topical application of estrogens may, in some cases, ameliorate lubrication.

4.2. Gastrointestinal Disorders. Constipation, and less frequently, incontinence of feces are common symptoms reported to the general practitioner, even more so by patients in advancing years. Disordered bowel function is a typical sequela of pontine and spinal lesions of the nervous system. In these cases, concomitant sensorimotor disturbances are usually present in addition to damaged autonomic fibers. So this permits data on the prevalence of gastrointestinal disorders in MS patients. However, speculations prevail when it comes to establishing connections between symptoms and the underlying disease, or to identifying a specific site of lesion. On direct questioning, 36 to $54 \%$ of the MS patients complained of constipation [4]. The degree of constipation is often more severe than the subjective affliction. Symptoms are furthermore felt to be milder than those of bladder dysfunction which is usually present as well. It is quite remarkable that nearly as many patients also relate-at least temporary-fecal incontinence [27]. Constipation or anal incontinence is particularly common as an additional associated aspect of bladder dysfunction [28].

All MS patients complaining of constipation or nontransitory incontinence of feces in terms of defined criteria should be sent to a gastroenterologic and proctologic specialist for further evaluation. MS-induced constipation is likely in the presence of notable pyramidal tract lesion with spasticity due to general immobility, increased muscle tone of the pelvic floor and semiparalyzed abdominal muscles. A mechanism was described with paradoxical contractions 
of the pelvic floor comparable to corresponding bladder dysfunction [29]. No reliable data exist concerning the question as to whether disturbed afferences and lesions of other autonomic or somatosensory nerves may also be involved in causing MS-specific impairment of intestinal motility. Lesions of the brain stem, the vagal nucleus in particular, result in slowed-down motility of the entire upper gastrointestinal tract down to the left colonic flexure.

The diagnosis of functional intestinal trouble encompasses the aforementioned interdisciplinary clinical examinations as well as the determination of colonic transit time, anal manometry, defecography, and electromyography as needed. The aforenamed examinations can help MS patients to objectivate the symptoms they had reported in the first place [30]. Electromyographic examination of external anal sphincter can either furnish proof of increased muscle tone and increased reflex activation or rule out possible peripheral neurogenic lesions [31].

The therapeutic procedure to improve disordered intestinal motility is based on general and less on MS-specific approaches: enhanced physical activity in spite of disability, adequate fluid intake which makes the optimum management of usually concomitant bladder dysfunction a prerequisite, and dietary changes. In severe slow transit constipation, macrogol is a helpful tool; outlet constipation will respond to the administration of enemas. In patients seriously afflicted, the anorectum may have to be cleared digitally. Pronounced spasticity of the anal sphincter can be mitigated by injections of Botulinum toxin. Trials with loperamide or anticholinergic agents may be successful in individual cases. What is mostly needed is care by perineal pads, diapers, and other aids.

Within this context, brief mention should be made of a special form of constipation: Overflow incontinence. When the rectum of a severely constipated patient is crammed with fecal matter that he is unable to evacuate, he is going to produce a scybalum with consecutive impaction of the feces. Bacterial liquefaction of stools consecutively results in incontinence with passage of liquid stools. This condition requires treatment of constipation. Management for diarrhea would be utterly contraproductive. Digital rectal palpation usually points to the right direction when the patient's rectum is filled up with stools.

4.3. Cardiovascular Disorders. Symptoms indicative of characteristic cardiovascular disorder in MS rarely seem related. When questioned, however, many patients complain of poor physical fitness and early exhaustion, but they fail to mention symptoms of orthostatic dysregulation, neurocardiogenic syncopes, or cardiac dysrhythmias. Through autonomic functional diagnostic workup in adequately equipped laboratories, a few work groups nonetheless succeeded in proving rather frequent disorders of the cardiovascular system. Older studies [32] as well as more recent publications [33] concurred in observing no abnormalities on orthostatic tests. Some other work groups though found orthostatic dysregulations in up to $25 \%$ of the MS patients [34, 35]. Similar results hold true for clinical symptoms. One should note that orthostatic dysregulation-the cardinal symptom of many autonomic disorders-never comes as the first manifestation or episode equivalent of multiple sclerosis. Even in the late stage, it is at most seen in a small group of patients.

Several work groups conducted a standardized series of parasympathetic and sympathetic tests in selected or consecutive groups of patients, and partly controls. Unfortunately, this study was originally designed to look at peripheral autonomic disorders (diabetic polyneuropathy), and thus not validated for central autonomic disorders. The results and correlations disclosed do not make for a uniform picture: Merkelbach and coworkers found hand grip test $(43 \%$ of the patients examined) to be the most conspicuous test suggesting sympathetic dysfunction. A difference between the varied courses of disease was not seen. McDougall reported the 30:15 ratio-and thus a mostly parasympathetic test in 16\% of his patients-being the most frequent pathology. De Seze cites blood pressure monitoring in orthostasis - a test of sympathetic function-in $18 \%$ as the most common remarkable feature that correlates significantly with a primary or secondary chronic course of disease. It was merely in Valsalva's maneuver-a largely parasympathetic test-that Flachenecker [36] found a major difference between the results for active and inactive disease.

There are no specific approaches to treat the rare orthostatic problems in MS. Basic management consists in adequate fluid intake and a diet rich in sodium. Patients will also benefit from wearing elastic stockings. Medication is useful to increase blood volume and the peripheral vessels tone, for instance by using fludrocortisone or a sympathomimetic.

4.4. Other Dysfunctions of the Autonomic System. The main sympathetic skin response was studied in various groups of MS patients. Several work groups [33, 35, 37] reported pathologic results in 40 to $50 \%$ of the patients. They described both, missing and delayed response, with leg leads being the chief provider of pathologic results. Abnormal measurements (in up to 94\%) were scarcely recorded [38]. Clinical symptoms regarding autonomic disturbances were not always related synchronously. A positive correlation with the duration of illness was seen in some patient groups, in others this pertained to the MS-induced disabilities.

Only a few studies used pupillometric tests in MS patients. The charming idea of observing a control system with sympathetic and parasympathetic impacts on pupil size is limited by the fact that many patients have a history of optic neuritis, which is likely to disrupt physiologic regulation later on. These measurements have failed to establish a uniform cardinal parameter. The conclusions drawn from them are incongruous [39].

Older publications claimed that hypo- or even anhidrosis of the lower limbs was the most common disturbance in thermoregulatory sweating in MS patients [40]. Patients in advanced stages of the disease are generally stricken by considerable spinal affliction.

Some authors [41] describe positive correlation between sympathetic vasomotor dysfunction and fatigue in MS 
patients. These observations have not been confirmed by larger studies and their contribution to the understanding of fatigue in MS remains uncertain.

\section{References}

[1] S. Poser, W. Poser, and G. Schlaf, "Prognostic indicators in multiple sclerosis," Acta Neurologica Scandinavica, vol. 74, no. 5, pp. 387-392, 1986.

[2] S. Beer and J. Kesselring, "Die multiple sklerose im kanton bern," Fortschritte der Neurologie Psychiatrie, vol. 56, no. 12, pp. 390-397, 1988.

[3] C. M. Poser, D. W. Paty, L. C. Scheinberg, W. I. McDonald, and G. C. Ebers, The Diagnosis of Multiple Sclerosis, ThiemeStratton, New York, Ny, USA, 1984.

[4] A. Hennessey, N. P. Robertson, R. Swingler, and D. A. S. Compston, "Urinary, faecal and sexual dysfunction in patients with multiple sclerosis," Journal of Neurology, vol. 246, no. 11, pp. 1027-1032, 1999.

[5] N. Kale, M. Setty, A. Jale, and T . Osman, "Assessment of autonomic nervous system dysfunction in multiple sclerosis and the association with clinical disability," Neurology International, vol. 1, article e5, 2009.

[6] R. A. Marrie, G. Cutter, T. Tyry, T. Vollmer, and D. Campagnolo, "Disparities in the management of multiple sclerosisrelated bladder symptoms," Neurology, vol. 68, no. 23, pp. 1971-1978, 2007.

[7] F. Patti, B. Ventimiglia, G. Failla, A. A. Genazzani, and A. Reggio, "Micturition disorders in multiple sclerosis patients: neurological, neurourodynamic and magnetic resonance findings," European Journal of Neurology, vol. 4, no. 3, pp. 259-265, 1997.

[8] C. Pozzilli, M. G. Grasso, S. Bastianello et al., "Structural brain correlates of neurourologic abnormalities in multiple sclerosis," European Neurology, vol. 32, no. 4, pp. 228-230, 1992.

[9] C. D. Betts, M. T. D’Mellow, and C. J. Fowler, "Urinary symptoms and the neurological features of bladder dysfunction in multiple sclerosis," Journal of Neurology Neurosurgery and Psychiatry, vol. 56, no. 3, pp. 245-250, 1993.

[10] M. B. Chancellor and J. G. Blaivas, "Urological and sexual problems in multiple sclerosis," Clinical Neuroscience, vol. 2, no. 3-4, pp. 189-195, 1994.

[11] M. de Sèze, A. Ruffion, P. Denys, P. A. Joseph, and B. PerrouinVerbe, "The neurogenic bladder in multiple sclerosis: review of the literature and proposal of management guidelines," Multiple Sclerosis, vol. 13, no. 7, pp. 915-928, 2007.

[12] A. Reitz, M. Stöhrer, G. Kramer et al., "European experience of 200 cases treated with botulinum-A toxin injections into the detrusor muscle for urinary incontinence due to neurogenic detrusor overactivity," European Urology, vol. 45, no. 4, pp. 510-515, 2004.

[13] V. Kalsi, G. Gonzales, R. Popat et al., "Botulinum injections for the treatment of bladder symptoms of multiple sclerosis," Annals of Neurology, vol. 62, no. 5, pp. 452-457, 2007.

[14] M. Hohenfellner, S. E. Dahms, K. Matzel, and J. W. Thüroff, "Sacral neuromodulation of the urinary bladderSakrale neuromodulation der harnblase," Urologe-Ausgabe A, vol. 39, no. 1, pp. 55-63, 2000.

[15] F. Khan, J. F. Pallant, J. I. Pallant, C. Brand, and T. J. Kilpatrick, "A randomised controlled trial: outcomes of bladder rehabilitation in persons with multiple sclerosis," Journal of Neurology,
Neurosurgery and Psychiatry, vol. 81, no. 9, pp. 1033-1038, 2010.

[16] H. Miller, C. A. Simpson, and W. K. Yeates, "Bladder dysfunction in multiple sclerosis," British Medical Journal, vol. 1, no. 5445, pp. 1265-1269, 1965.

[17] H. G. Lilius, E. J. Valtonen, and J. Wikstrom, "Sexual problems in patients suffering from multiple sclerosis," Scandinavian Journal of Social Medicine, vol. 4, no. 1, pp. 41-44, 1976.

[18] J. M. Minderhoud, J. G. Leemhuis, and J. Kremer, "Sexual disturbances arising from multiple sclerosis," Acta Neurologica Scandinavica, vol. 70, no. 4, pp. 299-306, 1984.

[19] M. Zorzon, R. Zivadinov, A. Bosco et al., "Sexual dysfunction in multiple sclerosis: a case-control study. I. Frequency and comparison of groups," Multiple Sclerosis, vol. 5, no. 6, pp. 418-427, 1999.

[20] K. M. Beier, D. Goecker, S. Babinsky, and C. J. Ahlers, "Sexualitä und partnerschaft bei multipler sklerose-ergebnisse einer empirischen studie bei betroffenen und ihren partnern," Sexuologie, vol. 9, no. 1, pp. 4-22, 2002.

[21] D. Mattson, M. Petrie, D. K. Srivastava, and M. McDermott, "Multiple sclerosis: sexual dysfunction and its response to medications," Archives of Neurology, vol. 52, no. 9, pp. 862868, 1995.

[22] C. D. Betts, S. J. Jones, C. G. Fowler, and C. J. Fowler, "Erectile dysfunction in multiple sclerosis. Associated neurological and neurophysiological deficits, and treatment of the condition," Brain, vol. 117, part 6, pp. 1303-1310, 1994.

[23] D. Borello-France, W. Leng, M. O’Leary et al., "Bladder and sexual function among women with multiple sclerosis," Multiple Sclerosis, vol. 10, no. 4, pp. 455-461, 2004.

[24] I. Gruenwald, Y. Vardi, I. Gartman et al., "Sexual dysfunction in females with multiple sclerosis: quantitative sensory testing," Multiple Sclerosis, vol. 13, no. 1, pp. 95-105, 2007.

[25] Y. Seçıl, Y. Yetımalar, M. Gedizlioğlu et al., "Sexual dysfunction and sympathetic skin response recorded from the genital region in women with multiple sclerosis," Multiple Sclerosis, vol. 13, no. 6, pp. 742-748, 2007.

[26] C. Fowler, J. Miller, and M. Sharief, "Viagra (sildenafil citrate) for the treatment of erectile dysfunction in men with multiple sclerosis," Annals of Neurology, vol. 46, p. 497, 1999.

[27] J. P. Hinds, B. H. Eidelman, and A. Wald, "Prevalence of bowel dysfunction in multiple sclerosis. A population survey," Gastroenterology, vol. 98, no. 6, pp. 1538-1542, 1990.

[28] Y. W. Chia, C. J. Fowler, M. A. Kamm, M. M. Henry, M. C. Lemieux, and M. Swash, "Prevalence of bowel dysfunction in patients with multiple sclerosis and bladder dysfunction," Journal of Neurology, vol. 242, no. 2, pp. 105-108, 1995.

[29] Y. W. Chia, K. P. Gill, J. S. Jameson et al., "Paradoxical puborectalis contraction is a feature of constipation in patients with multiple sclerosis," Journal of Neurology Neurosurgery and Psychiatry, vol. 60, no. 1, pp. 31-35, 1996.

[30] J. Weber, P. Grise, and M. Roquebert, "Radiopaque markers transit and anorectal manometry in 16 patients with multiple sclerosis and urinary bladder dysfunction," Diseases of the Colon and Rectum, vol. 30, no. 2, pp. 95-100, 1987.

[31] W. H. Jost, B. Schrank, A. Herold, and O. Leiss, "Functional outlet obstruction: anismus, spastic pelvic floor syndrome, and dyscoordination of the voluntary sphincter muscles. Definition, diagnosis, and treatment from the neurologic point of view," Scandinavian Journal of Gastroenterology, vol. 34, no. 5, pp. 449-453, 1999.

[32] A. B. Sterman, P. K. Coyle, D. J. Panasci, and R. Grimson, "Disseminated abnormalities of cardiovascular autonomic 
functions in multiple sclerosis," Neurology, vol. 35, no. 11, pp. 1665-1668, 1985.

[33] A. J. McDougall and J. G. McLeod, "Autonomic nervous system function in multiple sclerosis," Journal of the Neurological Sciences, vol. 215, no. 1-2, pp. 79-85, 2003.

[34] S. Merkelbach, U. Dillmann, C. Kölmel, J. Holz, and M. Müller, "Cardiovascular autonomic dysregulation and fatigue in multiple sclerosis," Multiple Sclerosis, vol. 7, no. 5, pp. 320326, 2001.

[35] J. de Sèze, T. Stojkovic, J. Y. Gauvrit et al., "Autonomic dysfunction in multiple sclerosis: cervical spinal cord atrophy correlates," Journal of Neurology, vol. 248, no. 4, pp. 297-303, 2001.

[36] P. Flachenecker, K. Reiners, M. Krauser, A. Wolf, and K. V. Toyka, "Autonomic dysfunction in multiple sclerosis is related to disease activity and progression of disability," Multiple Sclerosis, vol. 7, no. 5, pp. 327-334, 2001.

[37] B. Nazliel, C. Irkeç, and B. Koçer, "The roles of blink reflex and sympathetic skin response in multiple sclerosis diagnosis," Multiple Sclerosis, vol. 8, no. 6, pp. 500-504, 2002.

[38] B. Elie and J. P. Louboutin, "Sympathetic skin response (SSR) is abnormal in multiple sclerosis," Muscle and Nerve, vol. 18, no. 2, pp. 185-189, 1995.

[39] G. Pozzessere, P. Rossi, E. Valle, C. P. Froio, A. F. G. Petrucci, and C. Morocutti Md, "Autonomic involvement in multiple sclerosis: a pupillometric study," Clinical Autonomic Research, vol. 7, no. 6, pp. 315-319, 1997.

[40] N. E. Cartlidge, "Autonomic function in multiple sclerosis," Brain, vol. 95, no. 4, pp. 661-664, 1972.

[41] P. Flachenecker, A. Rufer, I. Bihler et al., "Fatigue in MS is related to sympathetic vasomotor dysfunction," Neurology, vol. 61, no. 6, pp. 851-853, 2003. 


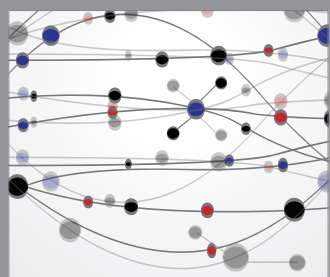

The Scientific World Journal
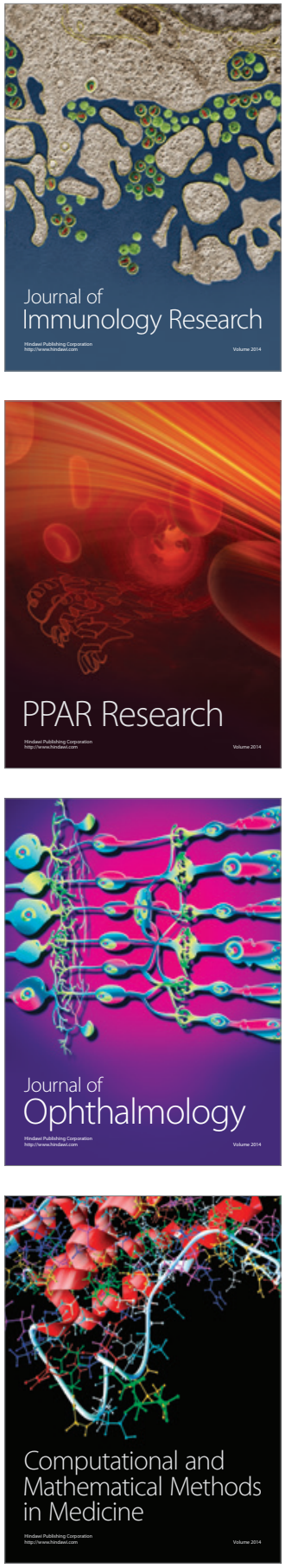

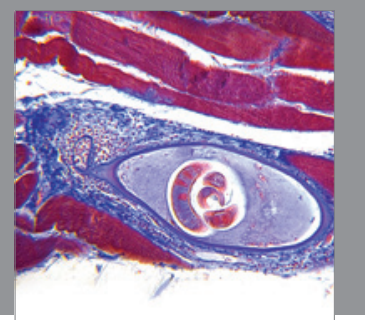

Gastroenterology

Research and Practice
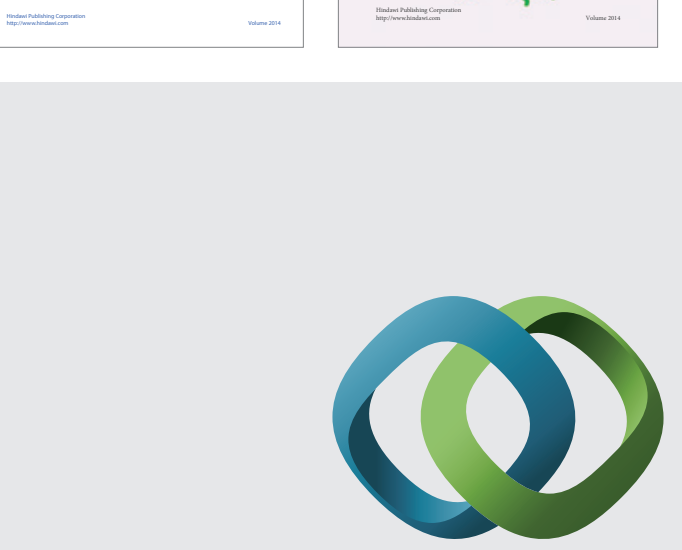

\section{Hindawi}

Submit your manuscripts at

http://www.hindawi.com
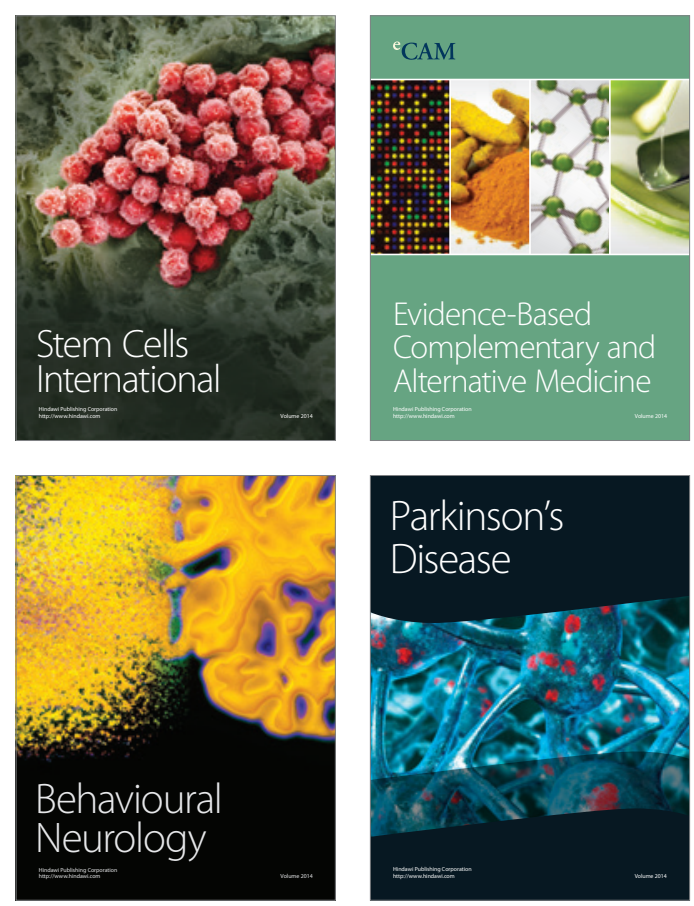

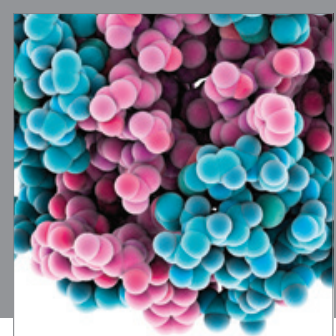

Journal of
Diabetes Research

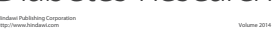

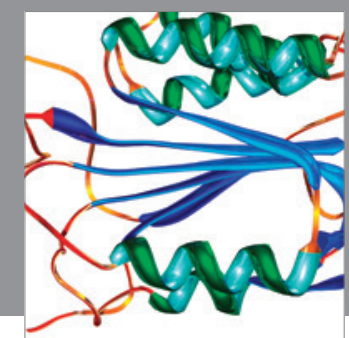

Disease Markers
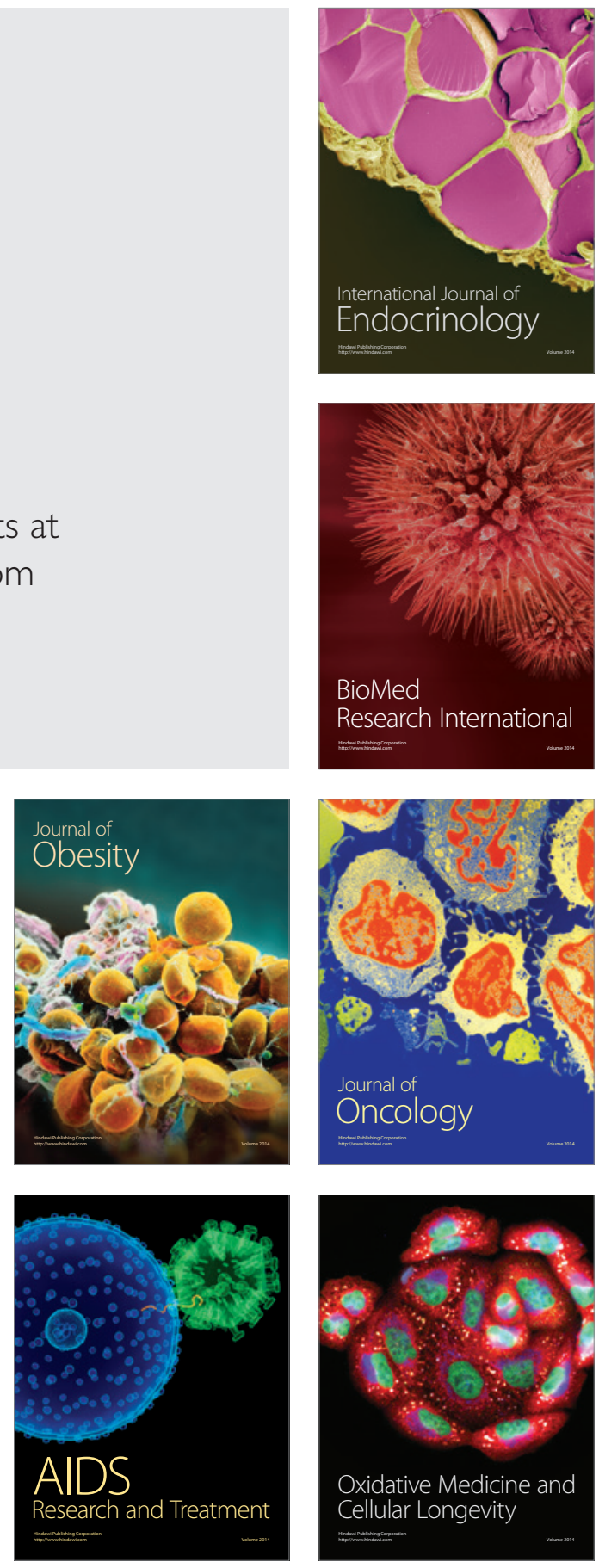